\title{
Surface-Enhanced Infrared Absorption of o-Nitroaniline on Nickel Nanoparticles Synthesized by Electrochemical Deposition
}

\author{
Yufang Niu, Jie Yao, and Keyu Wang \\ Analysis and Testing Center, Nanjing Normal University, Nanjing 210023, China \\ Correspondence should be addressed to Jie Yao; 07111@njnu.edu.cn
}

Received 21 January 2014; Revised 25 April 2014; Accepted 6 May 2014; Published 25 May 2014

Academic Editor: Sherine Obare

Copyright (C) 2014 Yufang Niu et al. This is an open access article distributed under the Creative Commons Attribution License, which permits unrestricted use, distribution, and reproduction in any medium, provided the original work is properly cited.

\begin{abstract}
Nickel nanoparticles were electrochemically deposited on indium-tin oxide (ITO) coated glass plate in a modified Watt's electrolyte. The surface-enhanced infrared absorption (SEIRA) effect of the nanoparticles was evaluated by attenuated total reflection spectroscopy (ATR-FTIR) using o-nitroaniline as a probe molecule. Electrodeposition parameters such as deposition time, $\mathrm{pH}$ value, and the type of surfactants were investigated. The morphology and the microstructure of the deposits were characterized by the field emission scanning electron microscope (FESEM) and the atomic force microscope (AFM), respectively. The results indicate that the optimum parameters were potential of $1.3 \mathrm{~V}$, time of $30 \mathrm{~s}$, and $\mathrm{pH}$ of 8.92 in the solution of $0.3756 \mathrm{~mol} / \mathrm{L}$ diethanolamine, $0.1 \mathrm{~mol} / \mathrm{L}$ nickel sulfate, $0.01 \mathrm{~mol} / \mathrm{L}$ nickel chloride, and $0.05 \mathrm{~mol} / \mathrm{L}$ boric acid. The FESEM observation shows that the morphology of nickel nanoparticles with best enhancement effect is spherical and narrowly distributed particles with the average size of $50 \mathrm{~nm}$. SEIRA enhancement factor is about 68 .
\end{abstract}

\section{Introduction}

When the molecules are adsorbed on nanoparticle metal films, the infrared absorption of molecules is enhanced, this phenomenon is called surface-enhanced infrared absorption (SEIRA) [1-3]. SEIRA can provide several orders of magnitude enhancement of modes in the IR region and has been successfully used in surface catalysis [4], analytical chemistry [5], biochemistry [6], sensor [7], metal corrosion [8], and surface photochemistry [9]. According to current research, the enhancement of IR is highly dependent on the morphology of the nanoparticles such as size [10], spacing [11], and thickness [12], as well as the polarization direction of incident light [13] and dielectric property of the metal [14]. Most of the studies show that $\mathrm{Au}$ and Ag exhibit SEIRA effect due to the stability and high enhanced factor but on the basis of electromagnetic mechanism, it is predicted that nearly all metals can yield the SEIRA effect $[3,15]$. Several papers have reported similar effects for metals except the coinage metals [16-20]. Nickel can be used to replace gold and silver because of the low cost. Williams et al. [21] prepared Ni microarray and found that the transmission spectra (FTIR) of alkyl mercaptan were significantly enhanced. Huo et al. [17] prepared Ni nanofilm on electrodes by potentiostatic electrodeposition method in the modified watt's electrolyte; the infrared absorption of probe molecules $\mathrm{CO}$ on Ni nanofilm exhibits inversed IR peak direction, significantly strengthened peak intensity, and widened half peak width. Therefore, $\mathrm{Ni}$ can be seen as a good SEIRA active substrate. However, to the best of our knowledge, there have not been any reports on SEIRA effect for nickel nanoparticles deposited on ITO substrate. So far, the use of electrochemical deposition has been considered as a practical and widely used method for nanoparticles preparation due to its ability to directly attach the nanoparticles on the substrate [22]. In the plating process, the morphology of the nanoparticles will be affected by factors such as the plating solution, the added agent (i.e., brightener [23], supporting electrolyte [24], surfactant, and so on), the $\mathrm{pH}$ value of the solution, the operating temperature, plating time, voltage, metal species, and so forth. The particle electrodeposition consists of two successive steps: (1) the transport of particles toward the surface which determines the surface coverage and (2) particle deposition onto the surface which determines the deposit morphology [25]. The mechanisms of the first step may be controlled by the electrophoretic force and are therefore correlated with the zeta potential of particles [26]. 
The mechanisms of the second step, determining the formation of particle aggregates, are more complex, as the morphology of the nanoparticle is closely related with enhancement effect. Preparation of controlled morphology of nanoparticles is a key part of a successful SEIRA experiment. The particle size and size distribution as well as stability of the system can all be controlled by adding surfactants. The surfactants have two functions: one is serving as a stabilizer, by complexing or coating ligand on the surface of particles to control the nucleation and growth of particles and the other is as a template agent; surfactant can be self-assembled to orderly structures in solvents such as micelles, liquid crystals, and vesicles, thus providing certain reaction space to obtain a controlled shape and particle size distribution of nanoparticles. Jana et al. [27] prepared gold nanorods in the presence of a rod-like micellar template, which is formed by cetyltrimethylammonium bromide (CTAB). The concentration of surfactant also has a great influence on the morphology of nanoparticle. Cha et al. [28] fabricated cobalt nanospheres, nanorods, nanoflowers, and nanoprisms by changing the amount of the surfactant and changing the morphology of the micelles. Lee et al. [29] have prepared cubic, cuboctahedral, and porous Pt nanoparticles using tetradecyltrimethylammonium bromide as a surface-stabilizing reagent by changing the $\mathrm{pH}$ value, which contributes to controlling the reaction rate. The results indicate that changing the $\mathrm{pH}$ value of the solution containing surfactant can also change the morphology of the prepared nanoparticles.

In this work, nickel film on ITO substrates was prepared by electrochemical deposition in a modified Watt's electrolyte; the surface-enhanced infrared effect of o-nitroaniline on it was measured by IR-ATR. The parameters' effect on the morphology of nanoparticles, such as deposition time, $\mathrm{pH}$ value, and the type of surfactants, was investigated. Finally the optimal preparation conditions were studied.

\section{Materials and Methods}

2.1. Materials. Hydrogen peroxide $\left(\mathrm{H}_{2} \mathrm{O}_{2}\right)$, ammonium hydroxide $\left(\mathrm{NH}_{3} \cdot \mathrm{H}_{2} \mathrm{O}\right)$, ethanol $\left(\mathrm{C}_{2} \mathrm{H}_{5} \mathrm{OH}\right)$, acetone $\left(\mathrm{C}_{3} \mathrm{H}_{6} \mathrm{O}\right)$, nickel sulfate $\left(\mathrm{NiSO}_{4}\right)$, nickel chloride $\left(\mathrm{NiCl}_{2}\right)$, boric acid $\left(\mathrm{H}_{3} \mathrm{BO}_{3}\right)$, diethanolamine, triethanolamine, polyethylene glycol 400, and o-nitroaniline were all of analytical grade. All chemicals were purchased from Sinopharm Chemical Reagent Co., Ltd. Distilled water was used during sample preparation. Indium-tin oxide (ITO) coated on glass plate with sheet resistance $25 \Omega$ was obtained from Conduc Optics and Electronics Co., Ltd.

2.2. Instruments. Surface morphology of the films was investigated with the field emission scanning electron microscope (FESEM, JSM-7600F electron microscope). Further microstructure analysis was carried out using atomic force microscope (AFM, Molecular Imaging PicoPlus). The chemical composition of the coatings was determined by an X-ray energy dispersive spectrometer (EDS, VANTAGE).

2.3. Substrate. Indium-tin oxide (ITO) coated on glass plate with sheet resistance of $25 \Omega$ was cleaned in acetone, ethanol, and deionized water with ultrasonic vibration for $10 \mathrm{~min}$. to remove lipid and other impurities and then boiled in mixed solution $\left(\mathrm{H}_{2} \mathrm{O}_{2}: \mathrm{NH}_{3} \cdot \mathrm{H}_{2} \mathrm{O}: \mathrm{H}_{2} \mathrm{O}=1: 1: 5, \mathrm{~V} / \mathrm{V}\right)$ to activate ITO substrate; finally the substrate was cleaned in deionized water with ultrasonic vibration for $10 \mathrm{~min}$. twice.

2.4. Electrochemical Deposition. Nickel films were electrodeposited from the electrolyte containing $0.1 \mathrm{~mol} / \mathrm{L}$ nickel sulfate, $0.01 \mathrm{~mol} / \mathrm{L}$ nickel chloride, $0.05 \mathrm{~mol} / \mathrm{L}$ boric acid, and $0.3756 \mathrm{~mol} / \mathrm{L}$ diethanolamine at the deposition potential of $1.3 \mathrm{~V}$. A potentiostat/galvanostat (CS150) was controlled with a computer potentiostatically. Moreover, all electrochemical measurements were carried out in a conventional threeelectrode system with ITO on glass plate as working electrode, platinum electrode as counter electrode, and saturated calomel electrode (SCE) as reference electrode. Deposition of the films was obtained at room temperature with magnetic stirring and the thickness of the deposits was controlled by time. After deposition, the substrates were rinsed and immersed in ethanol for $5 \mathrm{~min}$. to discontinue the reaction and to remove all unreacted electrolyte solution. The substrates were stored in desiccator for characterizations.

2.5. Adsorption of o-Nitroaniline. To investigate the SEIRA effect on nickel films synthesized by electrochemical deposition, $15 \mu \mathrm{L} 10^{-3} \mathrm{~mol} / \mathrm{L}$ o-nitroaniline/ethanol solution was dropped on the substrate to generate a self-assembled monolayer (SAM) on the Ni surface; the infrared spectra were collected after ethanol volatilization.

2.6. Spectra. The SEIRA spectra of o-nitroaniline on nickel films were recorded on Nicolet NEXUS 670 Fourier transform infrared (FT-IR) spectrometer in ATR mode, using a deuterated triglycine sulfate (DTGS) detector. All spectra were recorded with a resolution of $4 \mathrm{~cm}^{-1}$ and 32 scans in the spectral range between $650 \mathrm{~cm}^{-1}$ and $4000 \mathrm{~cm}^{-1}$. The background spectrum of atmosphere was measured as a single beam and was used as reference.

\section{Results and Discussion}

Figures 1(a) and 1(b) show infrared spectra of blank ITO glass and blank nickel films; there are no absorption peaks. Figures $1(\mathrm{c})$ and $1(\mathrm{~d})$ show infrared spectra of thin o-nitroaniline films of different concentrations dropped on blank ITO glass. When the concentration is $10^{-3} \mathrm{~mol} / \mathrm{L}$, the infrared spectrum cannot be detected. However, if $10^{-3} \mathrm{~mol} / \mathrm{L}$ o-nitroaniline is dropped on thin nickel films, the absorption signal increases significantly (see Figure 1(e)). The strong bands at $1345 \mathrm{~cm}^{-1}$ are assigned to the symmetric $\mathrm{NO}_{2}$ stretching mode. The $1512 \mathrm{~cm}^{-1}$ band is assigned to the antisymmetric $\mathrm{NO}_{2}$ stretching mode. In addition to these several bands of o-nitroaniline, the $\mathrm{NH}_{2}$ bending vibration mode of the molecule appears at $1629 \mathrm{~cm}^{-1}$; that is, there is no shifting in the positions of absorption peaks of o-nitroaniline. When the substrates are washed with ethanol, the peak intensity becomes weaker. The enhancement may be due to chemisorbed and physisorbed species. 


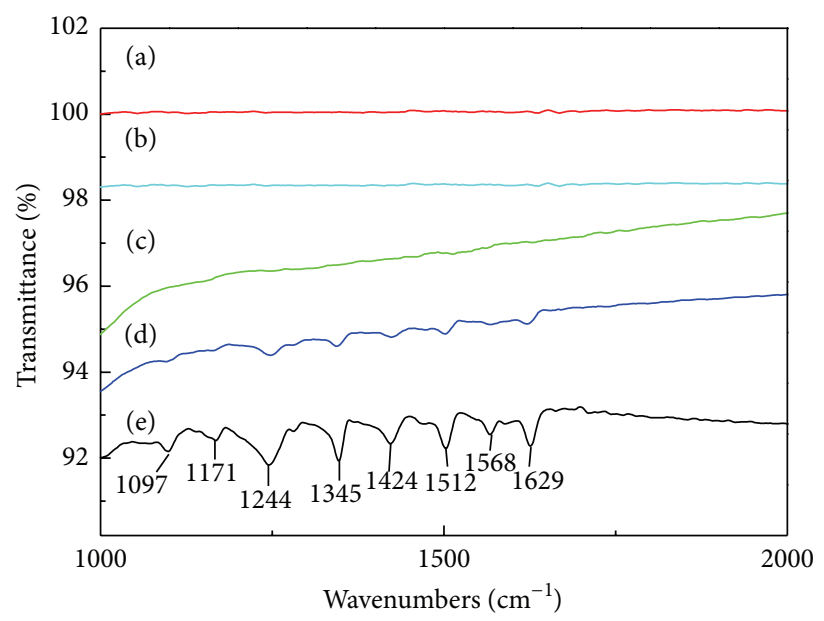

FIGURE 1: IR spectra of (a) blank ITO glass; (b) blank nickel films; (c) $10^{-3} \mathrm{~mol} / \mathrm{L}$ o-nitroaniline on ITO glass; (d) $10^{-2} \mathrm{~mol} / \mathrm{L}$ o-nitroaniline on ITO glass; and (e) $10^{-3} \mathrm{~mol} / \mathrm{L}$ o-nitroaniline on nickel films.

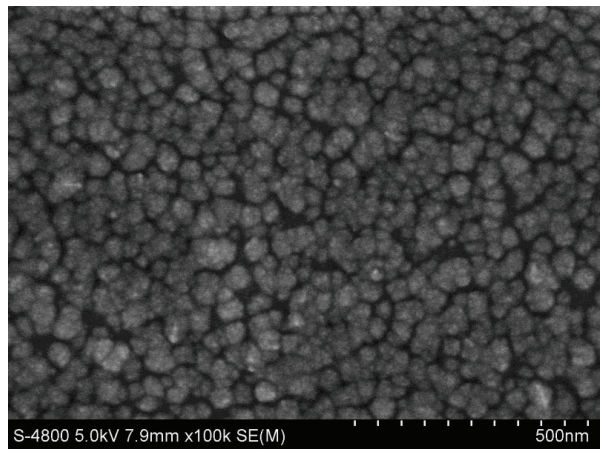

FIGURE 2: FESEM image of nickel nanoparticles electrodeposited on ITO glass substrate.

The o-nitroaniline infrared band at $1345 \mathrm{~cm}^{-1}$ is selected for evaluating the infrared enhancement factor. The surfaceenhancement factor (EF) is defined as

$$
\mathrm{EF}=\left(\frac{I_{\text {SEIRA }}}{I_{\text {infrared }}}\right)\left(\frac{M_{\text {bulk }}}{M_{\text {ads }}}\right),
$$

where $I_{\text {SEIRA }}$ and $I_{\text {infrared }}$ are the integrated infrared intensities of o-nitroaniline on SEIRA substrate and normal substrate, respectively. $M_{\text {bulk }}$ and $M_{\text {ads }}$ are the mass of o-nitroaniline molecules on normal substrate and SEIRA substrate, respectively. From the infrared spectra shown in Figure $1, I_{\text {infrared }}=$ $1, I_{\text {SEIRA }}=6.8, M_{\text {bulk }}=2.072 \times 10^{-5} \mathrm{~g}$, and $M_{\text {ads }}=2.072 \times$ $10^{-6} \mathrm{~g}$. A surface-enhancement factor of about 68 can be estimated.

Raman spectrum of o-nitroaniline on active substrate was also investigated. The result shows no characteristic peaks of o-nitroaniline. This can be interpreted that the thickness of the nanofilm with enhancement effect is different for SEIRA and SERS. Although Raman and infrared spectroscopy all belong to the molecular vibrational spectra, the former is the scattering spectrum and the latter is absorption spectrum. Light energy needed to produce Raman spectra is in the UV, visible, and near infrared and that of infrared spectroscopy is in the infrared. SERS, as a short-range effect, has been shown to be usually the most sensitive to the first adsorbed monolayer; the thickness with large SERS enhancement factor is no more than $800 \mathrm{~nm}$ [30-32]. On the other hand, SEIRA has a longer-range effect, which can frequently provide the enhancement of both the monolayer and multilayer; the thickness higher than $5 \mathrm{~nm}$ can exhibit SEIRA [12]. In our experiments, the thickness of Ni film is $2.5 \mu \mathrm{m}$, which is suitable for SEIRA but not suitable for SERS.

The surface morphology of the coatings was investigated by FESEM. Figure 2 shows FESEM image of nickel nanoparticles with best enhanced effect, showing that a large quantity of spherical particles with a narrow size distribution was achieved. And the spherical shaped particles possess an average diameter of about $50 \mathrm{~nm}$, and the spacing between the particles is approximately $5 \mathrm{~nm}$.

Figure 3 shows the AFM images of nickel nanoparticles electrodeposited on ITO glass substrate and the corresponding section analysis. It is clear that the surface is rough and the height of the particles protrusions is consistent. Based on the FESEM and AFM images, it is clear that nickel nanoparticles were narrowly distributed on the surface of the ITO glass, which is very important to the preparation of active substrate with enhanced effect. 

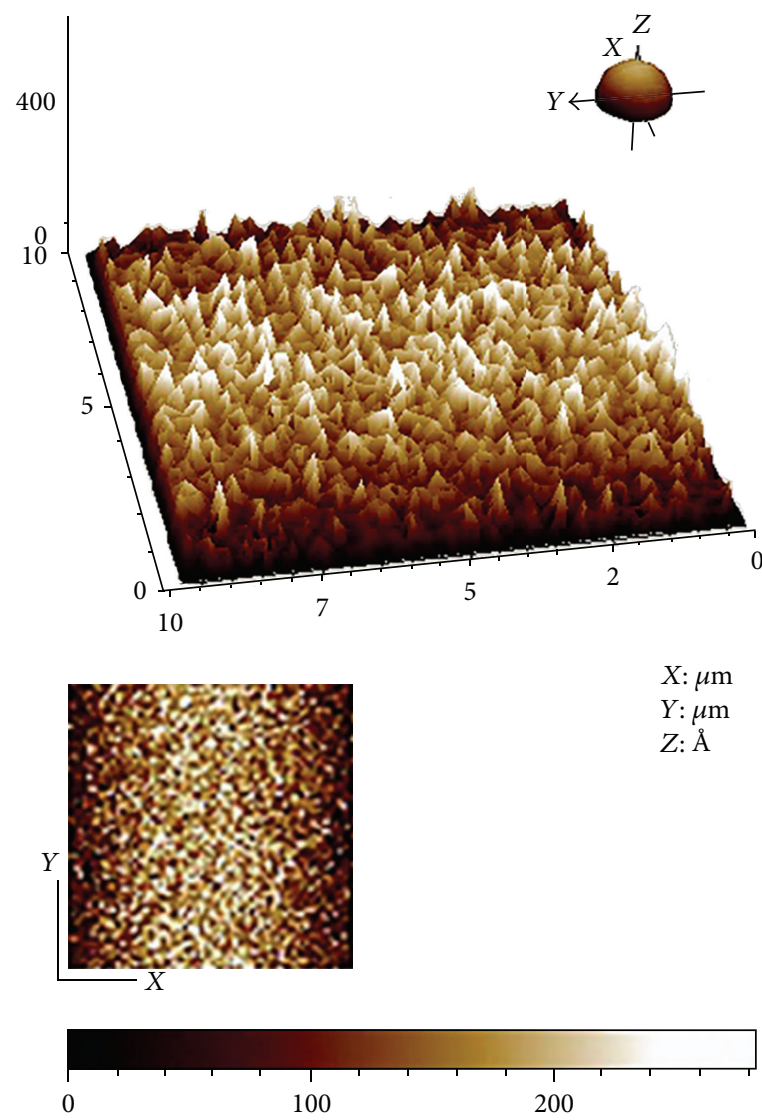

$(\AA)$

(a)
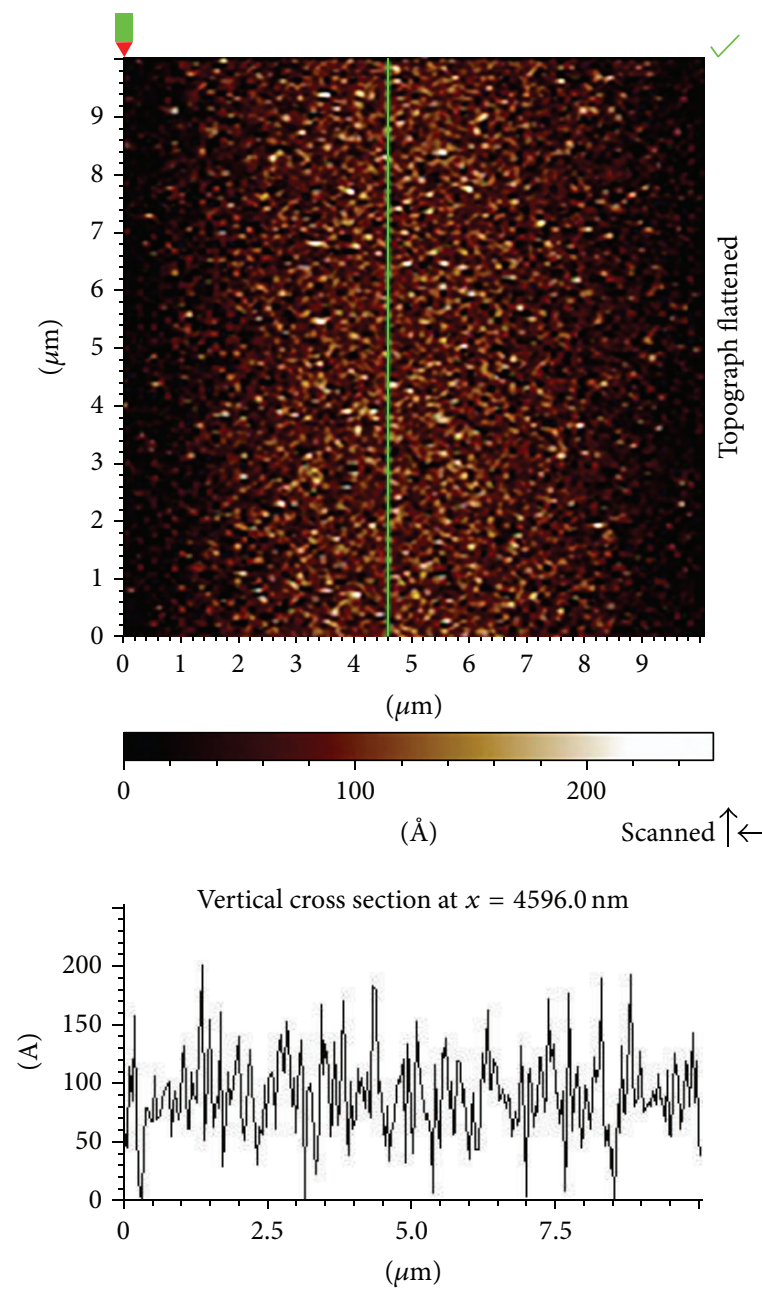

(b)

FIGURE 3: AFM images of nickel nanoparticles electrodeposited on ITO glass substrate: (a) 3D; (b) topograph flattened.

Figure 4 shows typical EDS spectrum of the electrodeposited nanoparticles prepared in the presence of $0.1 \mathrm{~mol} / \mathrm{L}$ nickel sulfate, $0.01 \mathrm{~mol} / \mathrm{L}$ nickel chloride, $0.05 \mathrm{~mol} / \mathrm{L}$ boric acid, and $0.3756 \mathrm{~mol} / \mathrm{L}$ diethanolamine with deposition time of $30 \mathrm{~s}$ and $\mathrm{pH}$ value of 8.92 . It can be proved that the $\mathrm{Ni}$ nanoparticles are successfully formed on the ITO surface. According to the linear scan voltammetry, when the voltage is $1.3 \mathrm{~V}$, metal nickel has not been oxidized, which further confirms that nickel is deposited on the substrate. Other peaks such as $\mathrm{In}, \mathrm{Si}, \mathrm{O}$, and $\mathrm{Na}$ are also observed. They refer to the component of ITO on glass plate.

The thickness and roughness of films vary with the time of deposition. They will affect the infrared enhancement effect. Liberman et al. [12] studied absorption spectrum of the silk protein at different thicknesses of Au films; the result shows that the enhancement varies with the thickness; after the film thickness becomes less than $5 \mathrm{~nm}$, one almost cannot see the SEIRA. In our experiment, thickness of Ni film is $2.5 \mu \mathrm{m}$, which is suitable for IR-ATR measurement.
To assess the effect of the deposition time on SEIRA spectrum of the adsorbed o-nitroaniline, the time of deposition was varied from $10 \mathrm{~s}$ to $120 \mathrm{~s}$. The SEIRA effect varies significantly depending on the deposition time as shown in Figure 5. At first with the increase of deposition time, SEIRA effect gradually increases. At $30 \mathrm{~s}$, the peak intensity of the probe molecule reaches the maximum, and then as time continues to increase, the peaks disappear gradually, which may be due to the fact that the long deposition time leads to the thickness of films beyond the scope of active substrate.

Figure 6(b) shows that when $0.3756 \mathrm{~mol} / \mathrm{L}$ diethanolamine is used as the surfactant, the nickel nanoparticles are spherical and narrowly distributed with the average particle size of $50 \mathrm{~nm}$; spacing between the particles is approximately $5 \mathrm{~nm}$. A controlled experiment was carried out in the absence of surfactant with other conditions unchanged. The nickel nanoparticles show irregular morphology consisting of nanosheets and nanospheres formed by the aggregation in Figure 6(a). It is clear that the addition of the surfactant can 


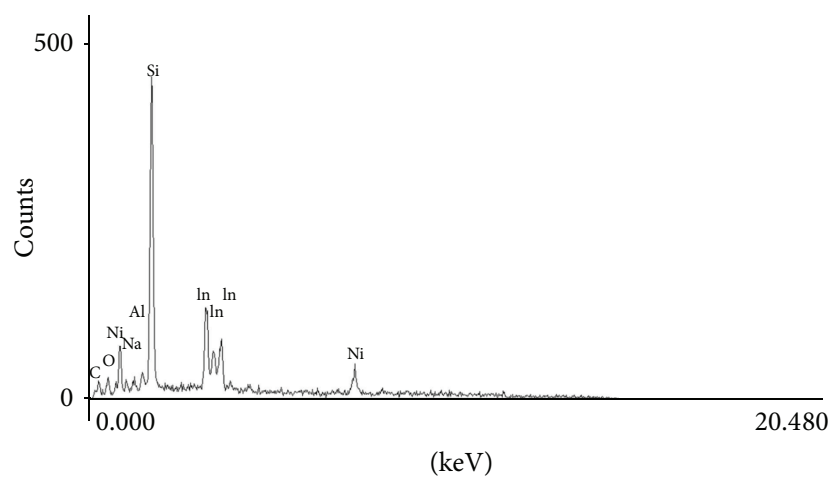

FIGURE 4: EDS spectrum of nickel nanoparticles electrodeposited on ITO glass substrate.

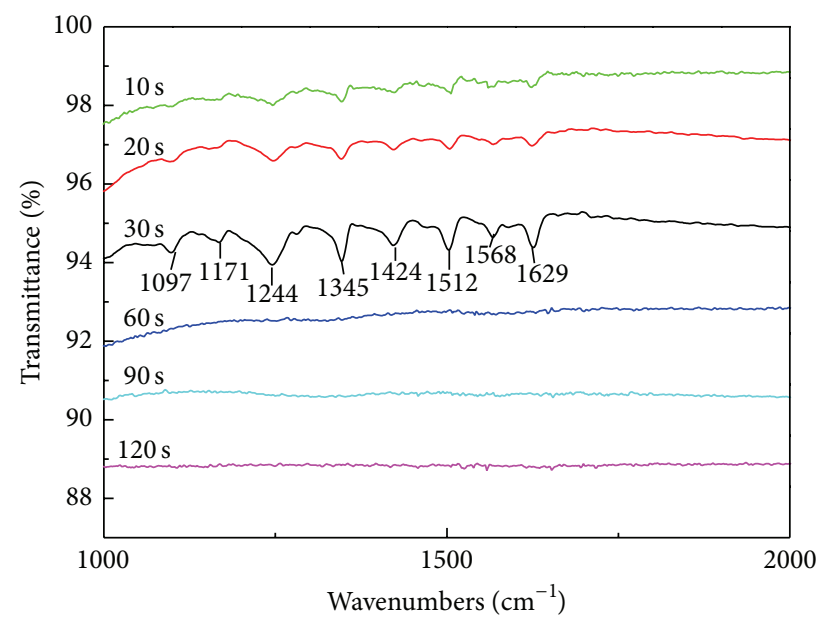

FIGURE 5: IR spectra of $10^{-3} \mathrm{~mol} / \mathrm{L}$ o-nitroaniline on nickel nanoparticles fabricated with different times.

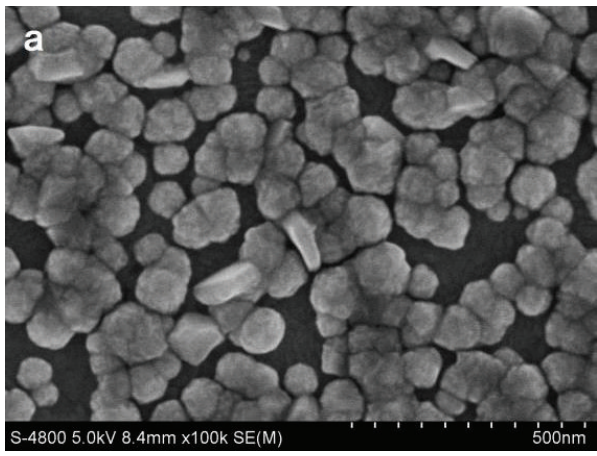

(a)

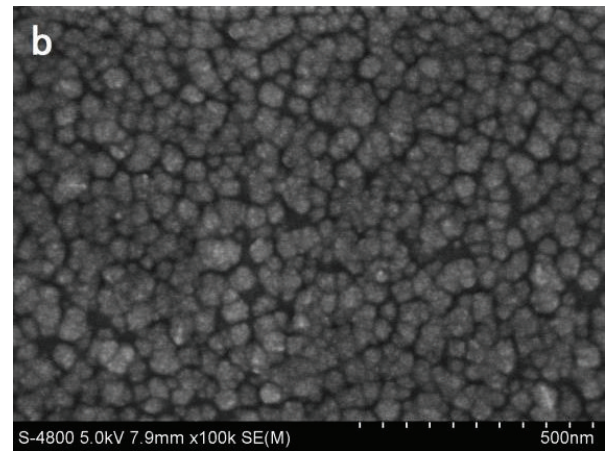

(b)

FIGURE 6: FESEM images of nickel nanoparticles fabricated (a) without diethanolamine and (b) with 0.3756 mol/L diethanolamine.

reduce aggregation of nickel nanoparticles and make them distribute narrowly. This may be related to the formation process of nickel crystal nucleus; in the absence of surfactant, the reaction between electrolyte generates nickel crystal nuclei first, and the crystal nuclei grow as the reaction continues, and ultimately irregular particles with severe aggregation. In the presence of diethanolamine, nickel ions first react with diethanolamine to form complex, afterward nickel crystal nuclei grow along a specific direction, which is controlled by the surfactant. So it can be concluded that the surfactant plays a crucial role in the morphology of nickel nanostructures. The surfactant as a structure-direct agent and dispersive agent can influence the growth of nickel nanostructures and as a stabilizer can prevent aggregation.

In order to investigate the effects of different surfactants on the morphology of nickel nanoparticles, 


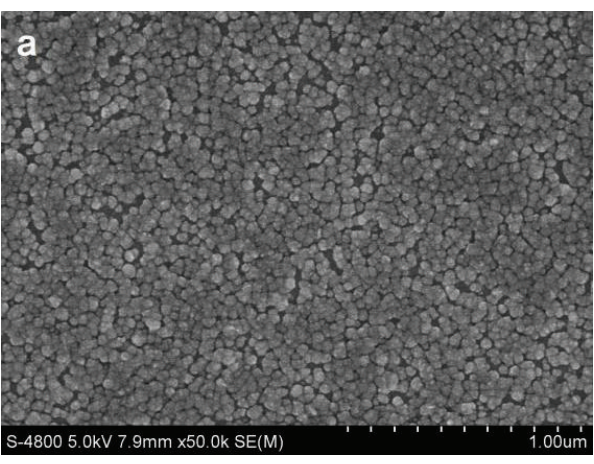

(a)

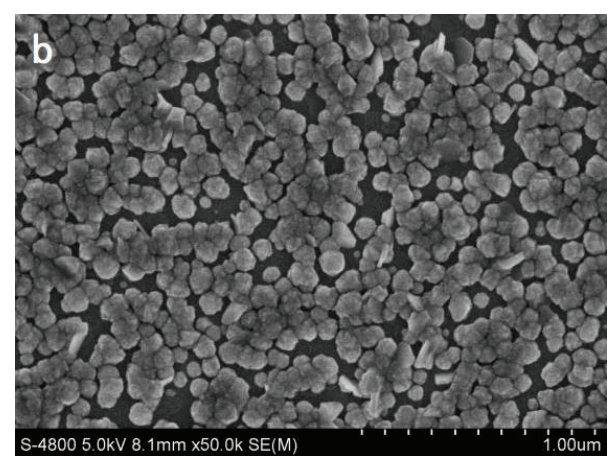

(b)

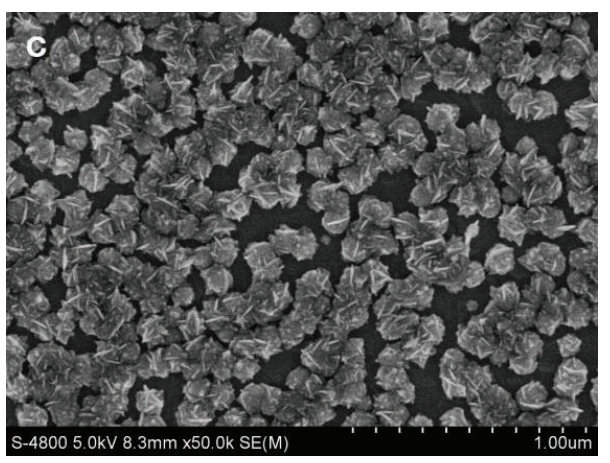

(c)

FIGURE 7: FESEM images of nickel nanoparticles fabricated with different surfactants (a) diethanolamine; (b) polyethylene glycol 400; and (c) triethanolamine.

triethanol-amine and polyethylene glycol 400 were used instead of diethanolamine in the same conditions. It is observed that nanoparticles exhibit irregular morphology consisting of nanospheres and multilateral morphology and are formed by the aggregation with polyethylene glycol 400 in Figure 7(b). It shows that polyethylene glycol 400 cannot control very well the nickel nanoparticles preparation. The FESEM image (Figure 7(c)) reveals that the shape of nickel nanoparticles is angular with size of about $100 \mathrm{~nm}$. This may be explained by the fact that these three surfactants all contain -OH groups, they are easy to form complexes with metal ions, to adsorb on the metal surface, and to control the nucleation and growth of metal particles, which affect the morphology of nickel nanoparticles. Due to difference of molecular structure, which contains different numbers of $-\mathrm{OH}$, the different surfactants lead to different morphologies.

IR spectra of $10^{-3} \mathrm{~mol} / \mathrm{L}$ o-nitroaniline on nickel nanoparticles fabricated with different surfactants are shown in Figure 8 . It can be seen that only nanoparticles fabricated with diethanolamine have enhanced effect. So it can be concluded that different kinds of surfactants have a great influence on the morphology of nanoparticles. Nanoparticles with SEIRA effect tend to be narrowly distributed with about the same size.

To assess the effect of the $\mathrm{pH}$ on the morphology of the nickel nanoparticles prepared with diethanolamine as the surfactant, the $\mathrm{pH}$ value is adjusted from 8.92 to 3.74 by $\mathrm{HCl}$. The morphology of the deposited nickel versus $\mathrm{pH}$ value is

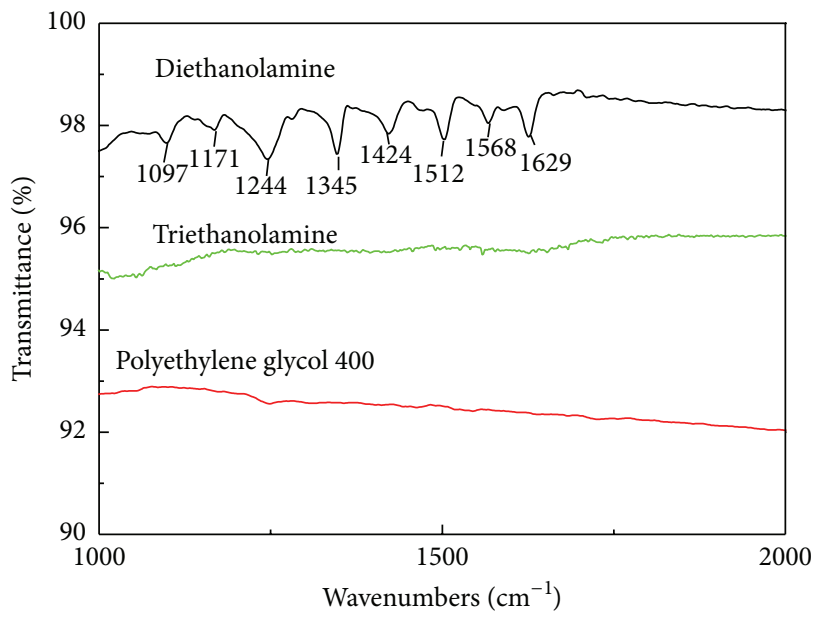

FIGURE 8: IR spectra of $10^{-3} \mathrm{~mol} / \mathrm{L}$ o-nitroaniline on nickel nanoparticles fabricated with different surfactants.

shown in Figure 9. When $\mathrm{pH}$ value is 8.92, nickel nanoparticles are spherical and narrowly distributed with the particle size of $50 \mathrm{~nm}$; spacing between the particles is approximately $5 \mathrm{~nm}$. With the $\mathrm{pH}$ value decreased, the density of the nanoparticles on the surface increases and the particle size and particle spacing become smaller. Only when the $\mathrm{pH}$ value is 5.35 , the gap of particles becomes slightly larger, which may be due to stacking. It may be that with the increasing amount of $\mathrm{HCl}$, the ionic strength increases, which may accelerate 


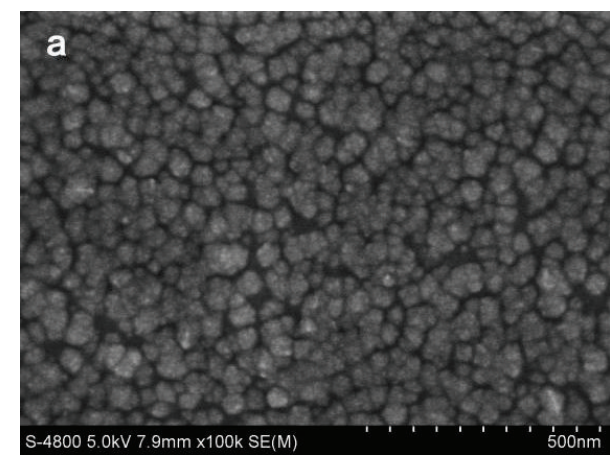

(a)

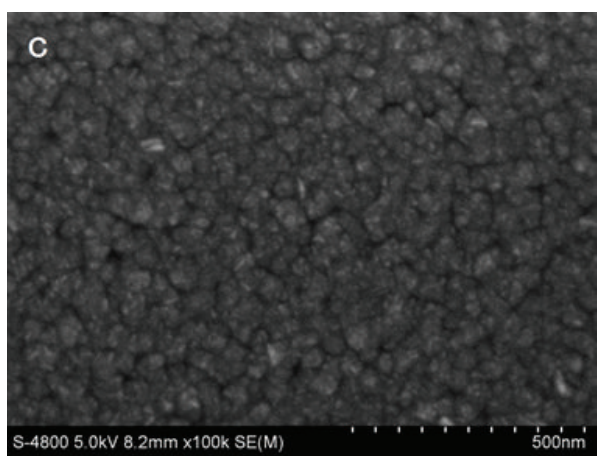

(c)

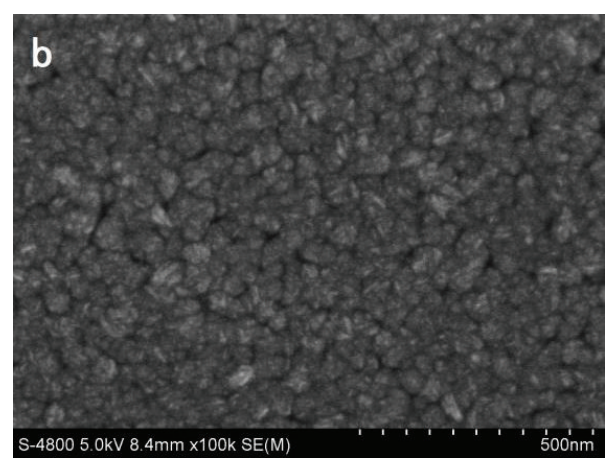

(b)

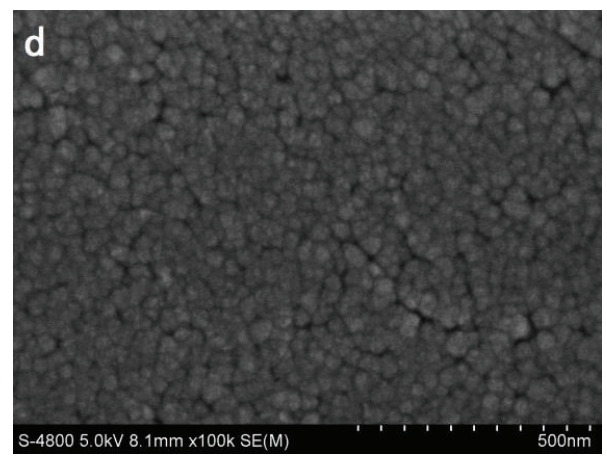

(d)

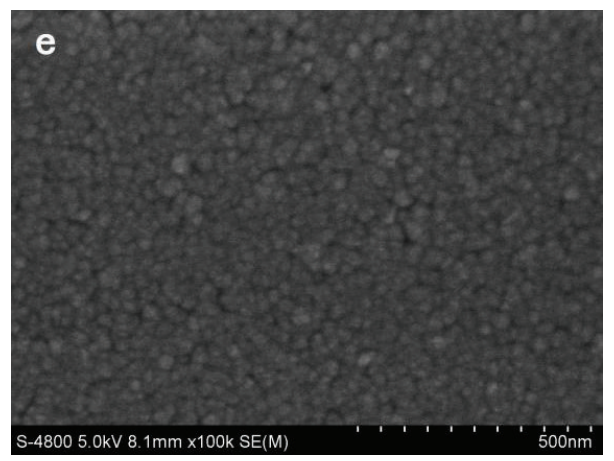

(e)

FIGURE 9: FESEM images of nickel nanoparticles fabricated with different pH values: (a) 8.92; (b) 7.68; (c) 6.79; (d) 5.35 ; and (e) 3.74 .

the reaction rate, and the hydrogen ions trigger cathodic reduction reaction leading to hydrogen evolution, thus accelerating the nucleation process. Therefore, the nucleation rate is greater than the growth rate, which increases the number of the seed and leads to smaller and more dense particle distribution.

When a monolayer of o-nitroaniline is bound to the surface of the nickel films, the intensity of the peaks in the o-nitroaniline spectrum becomes weaker with the decrease of the $\mathrm{pH}$ value and when it drops to 6.79 (see Figure 10), the peaks almost disappear. This may be explained by the fact that the particle spacing is related to the enhancement effect, which becomes weaker as the gap decreases. Therefore, nanoparticles with SEIRA effect tend to be as large as $50 \mathrm{~nm}$, and spacing between the particles is approximately $5 \mathrm{~nm}$.

Finally, we investigated active substrate's durability, nickel films were placed in a desiccator for 15 days, and then we recorded the infrared spectrum of the probe molecule. As shown in Figure 11, the peak strength of o-nitroaniline on freshly made nickel nanoparticles and on nickel nanoparticles after 15 days in desiccator is similar in enhancement effect, but the intensity of band at $1345 \mathrm{~cm}^{-1}$ decreases by about $30 \%$. Because the Ni films were slightly oxidized on the surface, it makes part of the surface unavailable to the o-nitroaniline adsorbate.

\section{Conclusions}

In this work, nickel nanoparticles on ITO glass were made by electrochemical deposition. The infrared spectra of monomolecular layers of $10^{-3} \mathrm{~mol} / \mathrm{L}$ o-nitroaniline adsorbed on thin nickel films were measured by ATR spectrum. The parameters' influence on SEIRA effect, such as time, $\mathrm{pH}$ value of solution, and surfactants was investigated and the optimal 


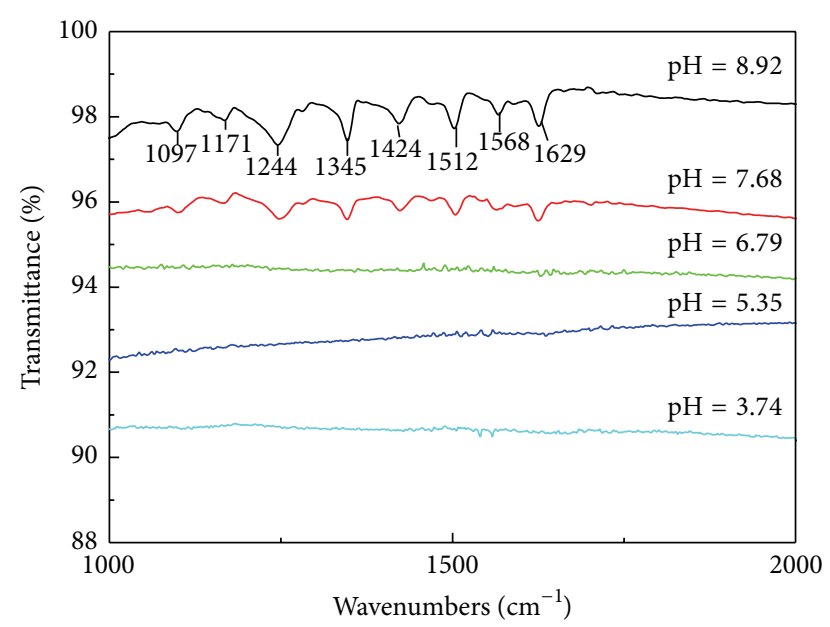

FIGURE 10: IR spectra of $10^{-3} \mathrm{~mol} / \mathrm{L}$ o-nitroaniline nickel nanoparticles fabricated with different $\mathrm{pH}$ values.

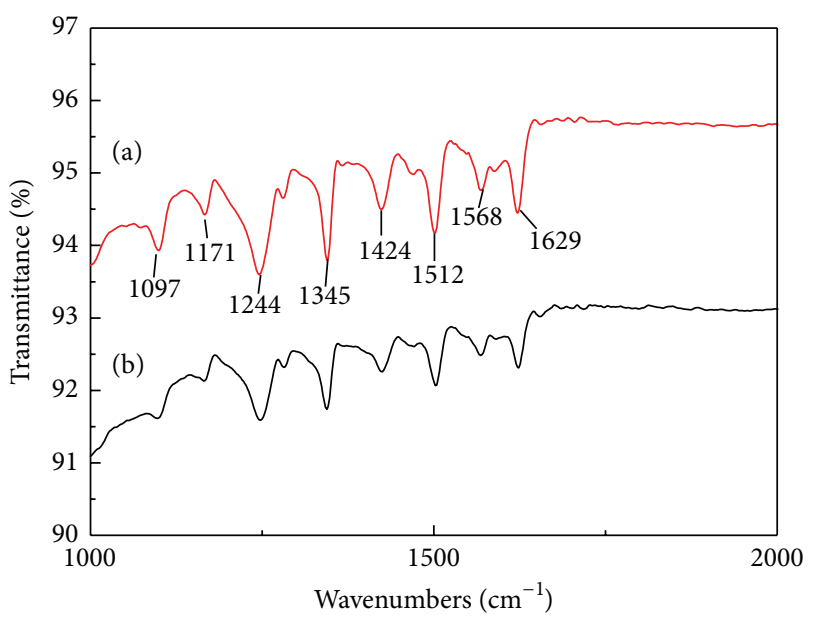

FIGURE 11: IR spectra of $10^{-3} \mathrm{~mol} / \mathrm{L}$ o-nitroaniline on (a) freshly prepared nickel nanoparticles and (b) nickel nanoparticles stored in desiccator for 15 days.

conditions were potential of $1.3 \mathrm{~V}$, time of $30 \mathrm{~s}$, and $\mathrm{pH}$ value of 8.92 in the solution of $0.3756 \mathrm{~mol} / \mathrm{L}$ diethanolamine. The FESEM image of nickel nanoparticles shows that the morphology with the best enhancement effect is spherical and narrowly distributed with the average particle size of $50 \mathrm{~nm}$, and spacing between the particles is approximately $5 \mathrm{~nm}$. SEIRA enhancement factor is about 68 .

\section{Conflict of Interests}

The authors declare that there is no conflict of interests regarding the publication of this paper.

\section{Acknowledgments}

The authors are grateful to Nanjing Normal University for providing the AFM and EDS for this work. The authors also sincerely thank Dr. Meng Yang and Professor Jialiang Wang for their help in the technical and language checking.

\section{References}

[1] R. F. Aroca, D. J. Ross, and C. Domingo, "Surface-enhanced infrared spectroscopy," Applied Spectroscopy, vol. 58, pp. 324A338A, 2004.

[2] M. Osawa and M. Ikeda, "Surface-enhanced infrared absorption of p-nitrobenzoic acid deposited on silver island films: contributions of electromagnetic and chemical mechanisms," The Journal of Physical Chemistry, vol. 95, no. 24, pp. 9914-9919, 1991.

[3] M. Osawa and B. Chem, "Dynamic processes in electrochemical reactions studied by surface-enhanced infrared absorption spectroscopy (SEIRAS)," Bulletin of the Chemical Society of Japan, vol. 70, no. 12, pp. 2861-2880, 1997.

[4] M. H. Shao, J. Warren, N. S. Marinkovic, P. W. Faguy, and R. R. Adzic, "In situ ATR-SEIRAS study of electrooxidation of dimethyl ether on a Pt electrode in acid solutions," Electrochemistry Communications, vol. 7, no. 5, pp. 459-465, 2005.

[5] H. D. W. Ck, B. Mizaikoff, N. Weissenbacher, and R. Kellner, "Surface enhanced infrared absorption spectroscopy (SEIRA) using external reflection on low-cost substrates," Fresenius' Journal of Analytical Chemistry, vol. 362, no. 1, pp. 15-20, 1998.

[6] C. S. Levin, J. Kundu, A. Barhoumi, and N. J. Halas, "Nanoshellbased substrates for surface enhanced spectroscopic detection of biomolecules," Analyst, vol. 134, no. 9, pp. 1745-1750, 2009.

[7] M. Fan, M. Thompson, M. L. Andrade, and A. G. Brolo, "Silver nanoparticles on a plastic platform for localized surface plasmon resonance biosensing," Analytical Chemistry, vol. 82, no. 15, pp. 6350-6352, 2010.

[8] K. P. Ishida and P. R. Griffiths, "Theoretical and experimental investigation of internal reflection at thin copper films exposed to aqueous solutions," Analytical Chemistry, vol. 66, no. 4, pp. 522-530, 1994.

[9] S. Sato, K. Ueda, Y. Kawasaki, and R. Nakamura, "In situ IR observation of surface species during the photocatalytic decomposition of acetic acid over $\mathrm{TiO}_{2}$ films," The Journal of Physical Chemistry B, vol. 106, no. 35, pp. 9054-9058, 2002.

[10] L. V. Brown, K. Zhao, N. King, H. Sobhani, P. Nordlander, and N. J. Halas, "Surface-enhanced infrared absorption using individual cross antennas tailored to chemical moieties," Journal of the American Chemical Society, vol. 135, no. 9, pp. 3688-3695, 2013.

[11] A. Pucci, F. Neubrech, D. Weber, S. Hong, T. Toury, and M. L. de la Chapelle, "Surface enhanced infrared spectroscopy using gold nanoantennas," Physica Status Solidi (B), vol. 247, no. 8, pp. 2071-2074, 2010.

[12] V. Liberman, R. Adato, T. H. Jeys, B. G. Saar, S. Erramilli, and H. Altug, "Rational design and optimization of plasmonic nanoarrays for surface enhanced infrared spectroscopy," Optics Express, vol. 20, no. 11, pp. 11953-11966, 2012.

[13] N. Berkovitch and M. Orenstein, "Thin wire shortening of plasmonic nanoparticle dimers: the reason for red shifts," Nano Letters, vol. 11, no. 5, pp. 2079-2082, 2011.

[14] Y.-K. Lin, H.-W. Ting, C.-Y. Wang et al., "Au nanocrystal array/silicon nanoantennas as wavelength-selective photoswitches," Nano Letters, vol. 13, no. 6, pp. 2723-2731, 2013.

[15] M. Osawa, K.-I. Ataka, K. Yoshii, and Y. Nishikawa, "Surfaceenhanced infrared spectroscopy: the origin of the absorption 
enhancement and band selection rule in the infrared spectra of molecules adsorbed on fine metal particles," Applied Spectroscopy, vol. 47, no. 9, pp. 1497-1502, 1993.

[16] C. K. Yee, R. Jordan, A. Ulman et al., "Novel one-phase synthesis of thiol-functionalized gold, palladium, and iridium nanoparticles using superhydride," Langmuir, vol. 15, no. 10, pp. 3486-3491, 1999.

[17] S. Huo, X. Xue, Y. Yan et al., "Extending in situ attenuated-totalreflection surface-enhanced infrared absorption spectroscopy to Ni electrodes," The Journal of Physical Chemistry B, vol. 110, no. 9, pp. 4162-4169, 2006.

[18] S. J. Huo, J. Y. Wang, J. L. Yao, and W. B. Cai, "Exploring electrosorption at iron electrode with in situ surface-enhanced infrared absorption spectroscopy," Analytical Chemistry, vol. 82, no. 12, pp. 5117-5124, 2010.

[19] L. A. Porter Jr., H. C. Choi, J. M. Schmeltzer, A. E. Ribbe, L. C. C. Elliott, and J. M. Buriak, "Electroless nanoparticle film deposition compatible with photolithography, microcontact printing, and dip-pen nanolithography patterning technologies," Nano Letters, vol. 2, no. 12, pp. 1369-1372, 2002.

[20] W. G. Lin, S. G. Sun, Z. Y. Zhou, S. P. Chen, and H. C. Wang, "Abnormal infrared effects of nanostructured rhodium thin films for CO adsorption at solid/gas interfaces," The Journal of Physical Chemistry B, vol. 106, no. 45, pp. 11778-11783, 2002.

[21] S. M. Williams, A. D. Stafford, K. R. Rodriguez, T. M. Rogers, and J. V. Coe, "Accessing surface plasmons with Ni microarrays for enhanced IR absorption by monolayers," The Journal of Physical Chemistry B, vol. 107, no. 43, pp. 11871-11879, 2003.

[22] M. S. El-Deab, "On the preferential crystallographic orientation of Au nanoparticles: effect of electrodeposition time," Electrochimica Acta, vol. 54, no. 14, pp. 3720-3725, 2009.

[23] H. P. Sachin, G. Achary, Y. Arthoba Naik, and T. V. Venkatesha, "Polynitroaniline as brightener for zinc-nickel alloy plating from non-cyanide sulphate bath," Bulletin of Materials Science, vol. 30, no. 1, pp. 57-63, 2007.

[24] L. J. Duić, Z. Mandić, and F. Kovačiček, "The effect of supporting electrolyte on the electrochemical synthesis, morphology, and conductivity of polyaniline," Journal of Polymer Science Part A: Polymer Chemistry, vol. 32, no. 1, pp. 105-111, 1994.

[25] C. Pignolet, M. Euvrard, A. Foissy, and C. Filiâtre, "Electrodeposition of latex particles in the presence of surfactant: investigation of deposit morphology," Journal of Colloid and Interface Science, vol. 349, no. 1, pp. 41-48, 2010.

[26] C. Filiâtre, C. Pignolet, A. Foissy, M. Zembala, and P. Warszyński, "Electrodeposition of particles at nickel electrode surface in a laminar flow cell," Colloids and Surfaces A: Physicochemical and Engineering Aspects, vol. 222, no. 1-3, pp. 55-63, 2003.

[27] N. R. Jana, L. Gearheart, and C. J. Murphy, "Wet chemical synthesis of high aspect ratio cylindrical gold nanorods," The Journal of Physical Chemistry B, vol. 105, no. 19, pp. 4065-4067, 2001.

[28] S. I. Cha, C. B. Mo, K. T. Kim, and S. H. Hong, "Ferromagnetic cobalt nanodots, nanorices, nanowires and nanoflowers by polyol process," Journal of Materials Research, vol. 20, no. 8, pp. 2148-2153, 2005.

[29] H. Lee, S. E. Habas, S. Kweskin, D. Butcher, G. A. Somorjai, and P. D. Yang, "Morphological control of catalytically active platinum nanocrystals," Angewandte Chemie-International Edition, vol. 45, no. 46, pp. 7824-7828, 2006.

[30] F. Yan, M. B. Wabuyele, G. D. Griffin, A. A. Vass, and T. Vo-Dinh, "Surface-enhanced Raman scattering detection of chemical and biological agent simulants,' IEEE Sensors Journal, vol. 5, no. 4, pp. 665-670, 2005.

[31] L. Baia, M. Baia, J. Popp, and S. Astilean, "Gold films deposited over regular arrays of polystyrene nanospheres as highly effective SERS substrates from visible to NIR," The Journal of Physical Chemistry B, vol. 110, no. 47, pp. 23982-23986, 2006.

[32] Y.-L. Deng and Y.-J. Juang, "Black silicon SERS substrate: effect of surface morphology on SERS detection and application of single algal cell analysis," Biosensors and Bioelectronics, vol. 53, no. 1, pp. 37-42, 2014. 

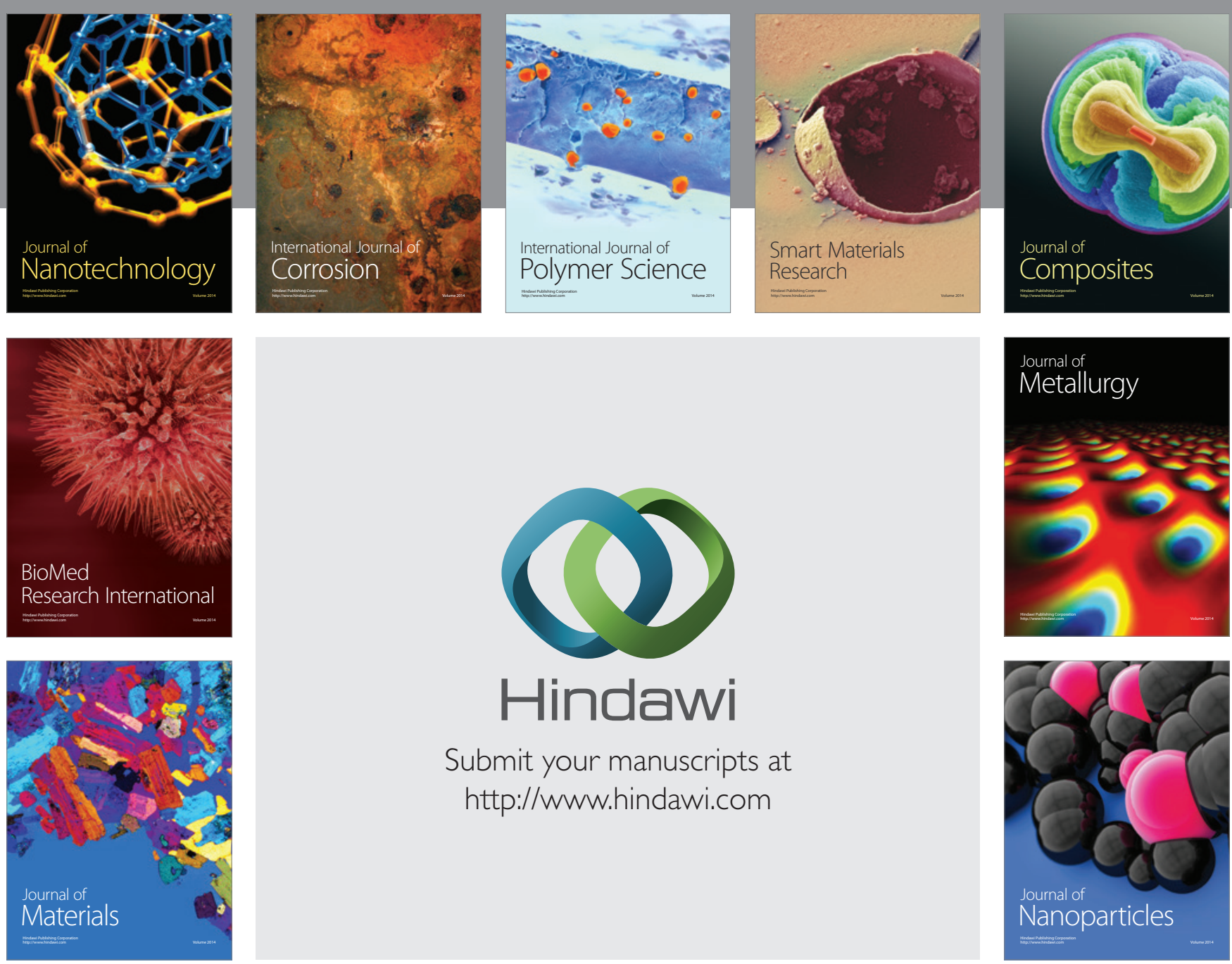

Submit your manuscripts at http://www.hindawi.com
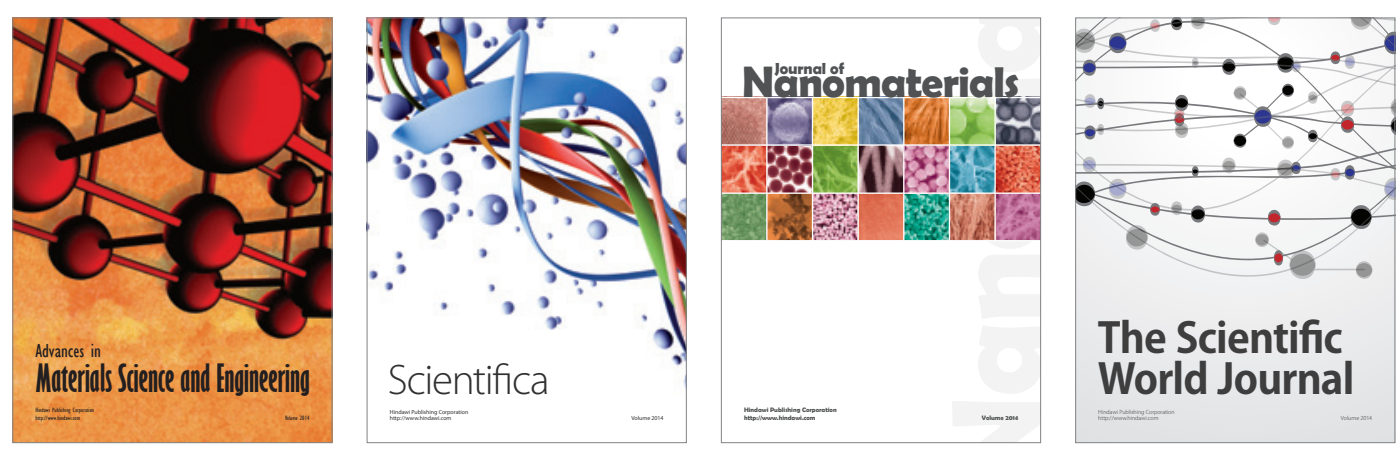

\section{The Scientific World Journal}
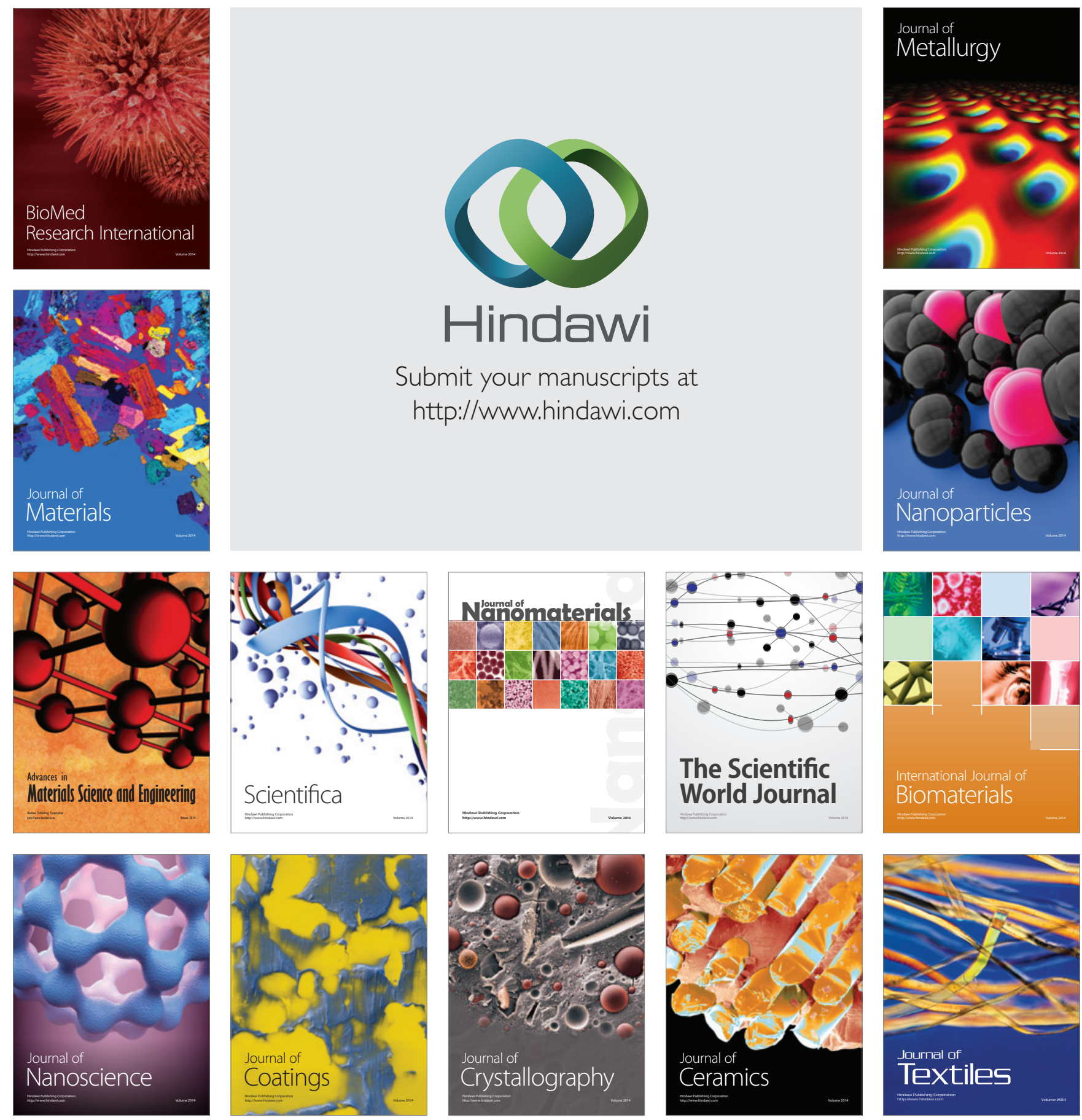